\title{
Practical Stability of Impulsive Discrete Systems with Time Delays
}

\author{
Liangji Sun, ${ }^{1}$ Chengyan Liu, ${ }^{2}$ and Xiaodi $\mathrm{Li}^{2}$ \\ ${ }^{1}$ Department of Computer Science, Shaanxi Vocational \& Technical College, Xi'an 710100, China \\ ${ }^{2}$ Department of Mathematics, Shandong Normal University, Jinan 250014, China
}

Correspondence should be addressed to Xiaodi Li; sodymath@163.com

Received 5 December 2013; Accepted 8 February 2014; Published 18 March 2014

Academic Editor: Haydar Akca

Copyright (c) 2014 Liangji Sun et al. This is an open access article distributed under the Creative Commons Attribution License, which permits unrestricted use, distribution, and reproduction in any medium, provided the original work is properly cited.

The purpose of this paper is to investigate the practical stability problem for impulsive discrete systems with time delays. By using Lyapunov functions and the Razumikhin-type technique, some criteria which guarantee the practical stability and uniformly asymptotically practical stability of the addressed systems are provided. Finally, two examples are presented to illustrate the criteria.

\section{Introduction}

As we all know, in many applications, we use discrete systems rather than continuous ones as the mathematical modeling, for example, numerical analysis, control theory, population models, and computer science [1-3]. Therefore, more and more attention has been paid to the theory of discrete systems, and some results for the stability of discrete systems have been obtained over the past few years [4-8].

The theory of practical stability has developed into a branch of the theory of motion stability [9]. Its notion is very useful, since it only needs to stabilize a system into a region of phase space. Based on this method, the desired state of a system can be unstable only if it oscillates sufficiently near this state. Recently, there has been a significant development in the theory of practical stability [10-15]. Moreover, impulses and time delays exist in many processes of dynamic systems, for example, physics, chemical technology, population dynamics, and neural networks, and they may impact systems seriously [16-30]. Therefore, it is necessary and important to analyze the practical stability of impulsive discrete systems with time delays.

In $[7,8]$, authors have obtained some results for asymptotic stability and exponential stability of impulsive discrete systems with time delays. Unfortunately, there is almost no result concerning uniformly asymptotically practical stability of impulsive discrete systems with time delays. The purpose of this paper is to establish some criteria which guarantee uniformly asymptotically practical stability of the addressed systems by using Lyapunov functions and the Razumikhin-type technique. This work is organized as follows. In Section 2, we introduce some basic definitions and notations. In Section 3, the main results are presented. In Section 4, two examples are discussed to illustrate the results.

\section{Preliminaries}

Let $\mathbb{R}_{+}$denote the set of nonnegative real numbers, $\mathbb{R}^{m}$ the $m$-dimensional real space equipped with the Euclidean norm $\|\cdot\|, \mathbb{Z}$ the set of integers, and $\mathbb{Z}_{+}$the set of positive integers. For any $r>0, r \in \mathbb{Z}_{+}, J \triangleq\{-r,-r+1,-r+2, \ldots,-1,0\}$, and set $C\left(\mathbb{R}_{+}, \mathbb{R}_{+}\right) \triangleq\left\{\phi: \mathbb{R}_{+} \rightarrow \mathbb{R}_{+} \mid \phi\right.$ is continuous $\}$. Let $S \triangleq\left\{\varphi: J \rightarrow \mathbb{R}^{m}\right\}$. Let $S_{\rho} \triangleq\{\varphi \in S:\|\varphi\|<\rho\}$. The norm of $\varphi$ is defined by $\|\varphi\|_{J}=\max _{s \in J}|\varphi(s)|$. The impulse times $n_{k}$ satisfy $0<n_{1}<n_{2}<\cdots<n_{k}<\cdots, n_{k}, k \in \mathbb{Z}_{+}$, and $\lim _{k \rightarrow+\infty} n_{k}=+\infty$.

Consider the following impulsive discrete systems with time delays:

$$
\begin{gathered}
x(n+1)=f\left(n, \bar{x}_{n}\right), \quad n \geq n_{0}, \quad n \in \mathbb{Z}_{+}, \\
\bar{x}(n)= \begin{cases}x(n), & n \neq n_{k}, k \in \mathbb{Z}_{+}, \\
x\left(n_{k}\right)+I_{k}\left(n_{k}, x\left(n_{k}\right)\right), & n=n_{k}, k \in \mathbb{Z}_{+},\end{cases} \\
x_{n_{0}}(s)=\varphi(s), \quad s \in J,
\end{gathered}
$$


where $0 \leq n_{0}<n_{1}, \varphi \in S, f \in \mathbb{Z}_{+} \times S_{\rho} \rightarrow \mathbb{R}^{m}, f(n, 0)=0$. For each $n \geq n_{0}, x_{n} \in S_{\rho}$ is defined by $x_{n}(s)=x(n+s), s \in J$. For each $k \in \mathbb{Z}_{+}, I_{k} \in \mathbb{Z}_{+} \times \mathbb{R}^{m} \rightarrow \mathbb{R}^{m}, I_{k}(n, 0)=0$, and, for any $\rho>0$, there exists a $\rho_{1} \in(0, \rho)$ such that $x \in S\left(\rho_{1}\right)$ implies that $x+I_{k} \in S(\rho)$.

In this paper, we assume that $f$ and $I_{k}$ satisfy certain conditions such that the solution of system (1) exists on $\left[n_{0}-\right.$ $r,+\infty) \cap \mathbb{Z}_{+}$and is unique [4]. We denote by $\bar{x}(n)=\bar{x}\left(n, n_{0}, \varphi\right)$ the solution of system (1) with initial value $\varphi$.

For convenience, we define the following classes of functions:

$K=\left\{w \in C\left(\mathbb{R}_{+}, \mathbb{R}_{+}\right): w\right.$ is strictly increasing and $w(0)=0\}$;

$K_{1}=\left\{w \in C\left(\mathbb{R}_{+}, \mathbb{R}_{+}\right): w(0)=0\right.$ and $w(s)>$ 0 for $s>0\}$;

$K_{2}=\left\{\psi \in C\left(\mathbb{R}_{+}, \mathbb{R}_{+}\right): \psi\right.$ is increasing and $\psi(s)<$ $s$ for $s>0\}$.

In addition, we introduce some definitions as follows.

Definition 1 (see [9]). Given two constants $\lambda$ and $A, 0<\lambda<$ $A$. Then, the impulsive discrete system (1) with respect to $(\lambda, A)$ is said to be

$\left(S_{1}\right)$ practically stable, if $\|\varphi\|_{J}<\lambda$ implies $\|x(n)\|<A, n \geq$ $n_{0}, n \in \mathbb{Z}_{+}$,

$\left(S_{2}\right)$ uniformly practically stable if $\left(S_{1}\right)$ holds, for every $n_{0} \in \mathbb{Z}_{+}$

$\left(S_{3}\right)$ asymptotically practically stable, if $\left(S_{1}\right)$ holds and, for any $\epsilon>0$, there exists $T=T\left(n_{0}, \epsilon\right)>0, T \in \mathbb{Z}_{+}$, such that $\|\varphi\|_{J}<\lambda$ implies $\|x(n)\|<\epsilon, n \geq n_{0}+T, n \in \mathbb{Z}_{+}$,

$\left(S_{4}\right)$ uniformly asymptotically practically stable if $\left(S_{2}\right)$ holds and the latter part of $\left(S_{3}\right)$ holds for a constant $T=T(\epsilon)>0, T \in \mathbb{Z}_{+}$, only dependent on $\epsilon$.

\section{Main Results}

Theorem 2. Assume that there exist functions $a, b \in K, \omega \in$ $C\left(\mathbb{R}_{+}, \mathbb{R}_{+}\right), \psi \in K_{2}, V: \mathbb{Z}_{+} \times \mathbb{R}^{m} \rightarrow \mathbb{R}_{+}$, such that

(i) $0<\lambda<A$ are given,

(ii) $a(\|x\|) \leq V(n, x) \leq b(\|x\|)$ for $(n, x) \in \mathbb{Z}_{+} \times \mathbb{R}^{m}$,

(iii) $V\left(n_{k}, \bar{x}\left(n_{k}\right)\right)=V\left(n_{k}, x\left(n_{k}\right)+I_{k}\left(n_{k}, x\left(n_{k}\right)\right)\right) \leq$ $\psi\left(V\left(n_{k}, x\left(n_{k}\right)\right)\right)$;

(iv) there is a function $P(s)$ continuous and nondecreasing for $s \geq 0$ and satisfying $P(s)>\psi^{-1}(s), s>0$, such that, for any solution $x(n)$ of system $(1), P(V(n, x(n))) \geq$ $V(n+s, x(n+s)), s \in J$, implies that

$$
\begin{aligned}
\Delta V(n, x(n)) & =V(n+1, x(n+1))-V(n, \bar{x}(n)) \\
& \leq \omega(V(n, \bar{x}(n))),
\end{aligned}
$$

where $\tau \triangleq \max _{k \in \mathbb{Z}_{+}}\left\{n_{k+1}-n_{k}\right\}$ and

$$
\tau \sup _{s>0} \frac{\omega(s)}{s}<1-2\left(\inf _{s>0} \frac{\psi^{-1}(s)}{s}\right)^{-1},
$$

(v) $b(\lambda)<\psi(a(A))$.
Then, the system (1) with respect to $(\lambda, A)$ is uniformly asymptotic practically stable.

Proof. Let

$$
q \triangleq \sup _{s>0} \frac{\omega(s)}{s}, \quad p \triangleq\left(\inf _{s>0} \frac{\psi^{-1}(s)}{s}\right)^{-1}<1
$$

For any $n_{0} \geq 0$, let $\bar{x}(n) \doteq \bar{x}\left(n, n_{0}, \varphi\right)$ be the solution of system (1) through $\left(n_{0}, \varphi\right)$, where $\left(n_{0}, \varphi\right) \in \mathbb{Z}_{+} \times S$, and $\|\varphi\|_{J}<\lambda$. It suffices to show that

$$
\|x\|<A, \quad n \geq n_{0}, \quad n \in \mathbb{Z}_{+} .
$$

Now, we show that

$$
V(n, x(n)) \leq \psi^{-1}(b(\lambda)), \quad n \in\left[n_{0}, n_{1}\right] \cap \mathbb{Z}_{+} .
$$

If it does not hold, then there exists a $r \in\left[n_{0}, n_{1}\right] \cap \mathbb{Z}_{+}$, such that $V(r, x(r))>\psi^{-1}(b(\lambda))$. Let $r_{2}=\min \{n: V(n, x(n))>$ $\left.\psi^{-1}(b(\lambda)), n \in\left[n_{0}, n_{1}\right] \cap \mathbb{Z}_{+}\right\}$. Since $V\left(n_{0}, x\left(n_{0}\right)\right) \leq b(\lambda) \leq$ $\psi^{-1}(b(\lambda))$, it is clear that $r_{2}>n_{0}$. Let $r_{1}=\max \{n$ : $\left.V(n, x(n)) \leq b(\lambda), n \in\left[n_{0}, r_{2}\right) \cap \mathbb{Z}_{+}\right\}$. Thus,

$$
\begin{gathered}
V\left(r_{2}, x\left(r_{2}\right)\right)>\psi^{-1}(b(\lambda)), \quad V\left(r_{1}, x\left(r_{1}\right)\right) \leq b(\lambda), \\
b(\lambda)<V(t, x(t)) \leq \psi^{-1}(b(\lambda)), \quad n \in\left(r_{1}, r_{2}\right) \cap \mathbb{Z}_{+} .
\end{gathered}
$$

Hence, we obtain

$$
\begin{aligned}
V\left(r_{2}, x\left(r_{2}\right)\right)-V\left(r_{1}, x\left(r_{1}\right)\right) & >\psi^{-1}(b(\lambda))-b(\lambda) \\
& =\psi^{-1}(b(\lambda))\left(1-\frac{b(\lambda)}{\psi^{-1}(b(\lambda))}\right) \\
& \geq \psi^{-1}(b(\lambda))(1-p) .
\end{aligned}
$$

By (7), we obtain that, for any $n \in\left[r_{1}, r_{2}\right] \cap \mathbb{Z}_{+}$,

$$
\begin{aligned}
P(V(n, x(n))) & >\psi^{-1}(V(n, x(n))) \geq \psi^{-1}(b(\lambda)) \\
& \geq V(n+s, x(n+s)), \quad s \in J .
\end{aligned}
$$


Using condition (iv), the inequality $\Delta V(n, x(n)) \leq$ $\omega(V(n, \bar{x}(n)))=\omega(V(n, x(n)))$ holds for all $n \in\left[r_{1}, r_{2}\right] \cap \mathbb{Z}_{+}$. Thus,

$$
\begin{aligned}
V\left(r_{2}, x(\right. & \left.\left.r_{2}\right)\right)-V\left(r_{1}, x\left(r_{1}\right)\right) \\
= & V\left(r_{2}, x\left(r_{2}\right)\right)-V\left(r_{2}-1, x\left(r_{2}-1\right)\right) \\
& +V\left(r_{2}-1, x\left(r_{2}-1\right)\right)-V\left(r_{2}-2, x\left(r_{2}-2\right)\right) \\
& +\cdots+V\left(r_{1}+1, x\left(r_{1}+1\right)\right)-V\left(r_{1}, x\left(r_{1}\right)\right) \\
= & \Delta V\left(r_{2}-1, x\left(r_{2}-1\right)\right)+\Delta V\left(r_{2}-2, x\left(r_{2}-2\right)\right) \\
& +\cdots+\Delta V\left(r_{1}, x\left(r_{1}\right)\right) \\
\leq & \omega\left(V\left(r_{2}-1, x\left(r_{2}-1\right)\right)\right)+\omega\left(V\left(r_{2}-2, x\left(r_{2}-2\right)\right)\right) \\
& +\cdots+\omega\left(V\left(r_{1}, x\left(r_{1}\right)\right)\right) \\
\leq & V\left(r_{2}-1, x\left(r_{2}-1\right)\right) \frac{\omega\left(V\left(r_{2}-1, x\left(r_{2}-1\right)\right)\right)}{V\left(r_{2}-1, x\left(r_{2}-1\right)\right)} \\
& +\cdots+V\left(r_{1}, x\left(r_{1}\right)\right) \frac{\omega\left(V\left(r_{1}, x\left(r_{1}\right)\right)\right)}{V\left(r_{1}, x\left(r_{1}\right)\right)} \\
\leq & \psi-1(b(\lambda)) q \tau .
\end{aligned}
$$

From (8) and (10), it can be deduced that $1-p<q \tau$, which is a contradiction with the condition (iv) and, thus, (6) holds.

Then, it follows from condition (iii) that

$$
\begin{aligned}
V\left(n_{1}, \bar{x}\left(n_{1}\right)\right) & =V\left(n_{1}, x\left(n_{1}\right)+I_{1}\left(n_{1}, x\left(n_{1}\right)\right)\right) \\
& \leq \psi\left(V\left(n_{1}, x\left(n_{1}\right)\right)\right) \leq b(\lambda) .
\end{aligned}
$$

Next, we claim that

$$
V(n, x(n)) \leq \psi^{-1}(b(\lambda)), \quad n \in\left[n_{1}, n_{2}\right] \cap \mathbb{Z}_{+} .
$$

If this assertion is not true, then there exists a $r \in$ $\left[n_{1}, n_{2}\right] \cap \mathbb{Z}_{+}$, such that $V(r, x(r))>\psi^{-1}(b(\lambda))$. Let $r_{4}=$ $\min \left\{n: V(n, x(n))>\psi^{-1}(b(\lambda)), n \in\left[n_{1}, n_{2}\right] \cap \mathbb{Z}_{+}\right\}$. Since $V\left(n_{1}, x\left(n_{1}\right)\right) \leq b(\lambda) \leq \psi^{-1}(b(\lambda))$, we have

$$
r_{4}>n_{1}, \quad V\left(r_{4}, x\left(r_{4}\right)\right)>\psi^{-1}(b(\lambda)) .
$$

Let $r_{3}=\max \left\{n: V(n, x(n)) \leq b(\lambda), n \in\left[n_{1}, r_{4}\right) \cap \mathbb{Z}_{+}\right\}$. Thus,

$$
\begin{gathered}
V\left(r_{3}, x\left(r_{3}\right)\right) \leq b(\lambda), \\
b(\lambda)<V(n, x(n)) \leq \psi^{-1}(b(\lambda)), \quad n \in\left(r_{3}, r_{4}\right) \cap \mathbb{Z}_{+} .
\end{gathered}
$$

Hence, we obtain

$$
\begin{aligned}
V\left(r_{4}, x\left(r_{4}\right)\right)-V\left(r_{3}, x\left(r_{3}\right)\right) & >\psi^{-1}(b(\lambda))-b(\lambda) \\
& =\psi^{-1}(b(\lambda))\left(1-\frac{b(\lambda)}{\psi^{-1}(b(\lambda))}\right) \\
& \geq \psi^{-1}(b(\lambda))(1-p) .
\end{aligned}
$$

Considering (15), we obtain, for any $n \in\left[r_{3}, r_{4}\right] \cap \mathbb{Z}_{+}$,

$$
\begin{aligned}
P(V(n, x(n))) & >\psi^{-1}(V(n, x(n))) \geq \psi^{-1}(b(\lambda)) \\
& \geq V(n+s, x(n+s)), \quad s \in J .
\end{aligned}
$$

Using condition (iv), the inequality $\Delta V(n, x(n)) \leq \omega(V(n$, $\bar{x}(n)))=\omega(V(n, x(n)))$ holds for all $n \in\left[r_{3}, r_{4}\right] \cap \mathbb{Z}_{+}$. Thus,

$$
\begin{aligned}
V\left(r_{4}, x(\right. & \left.\left.r_{4}\right)\right)-V\left(r_{3}, x\left(r_{3}\right)\right) \\
= & V\left(r_{4}, x\left(r_{4}\right)\right)-V\left(r_{4}-1, x\left(r_{4}-1\right)\right) \\
& +V\left(r_{4}-1, x\left(r_{4}-1\right)\right)-V\left(r_{4}-2, x\left(r_{4}-2\right)\right) \\
& +\cdots+V\left(r_{3}+1, x\left(r_{3}+1\right)\right)-V\left(r_{3}, x\left(r_{3}\right)\right) \\
= & \Delta V\left(r_{4}-1, x\left(r_{4}-1\right)\right)+\Delta V\left(r_{4}-2, x\left(r_{4}-2\right)\right) \\
& +\cdots+\Delta V\left(r_{3}, x\left(r_{3}\right)\right) \\
\leq & \omega\left(V\left(r_{4}-1, x\left(r_{4}-1\right)\right)\right)+\omega\left(V\left(r_{4}-2, x\left(r_{4}-2\right)\right)\right) \\
& +\cdots+\omega\left(V\left(r_{3}, x\left(r_{3}\right)\right)\right) \\
\leq & V\left(r_{4}-1, x\left(r_{4}-1\right)\right) \frac{\omega\left(V\left(r_{4}-1, x\left(r_{4}-1\right)\right)\right)}{V\left(r_{4}-1, x\left(r_{4}-1\right)\right)} \\
& +\cdots+V\left(r_{3}, x\left(r_{3}\right)\right) \frac{\omega\left(V\left(r_{3}, x\left(r_{3}\right)\right)\right)}{V\left(r_{3}, x\left(r_{3}\right)\right)} \\
\leq & \psi(b(\lambda)) q \tau .
\end{aligned}
$$

From (16) and (18), it can be deduced that $1-p<q \tau$, which is a contradiction with the condition (iv) and, thus, (12) holds.

Then, it follows from condition (iii) that

$$
\begin{aligned}
V\left(n_{2}, \bar{x}\left(n_{2}\right)\right) & =V\left(n_{2}, x\left(n_{2}\right)+I_{2}\left(n_{2}, x\left(n_{2}\right)\right)\right) \\
& \leq \psi\left(V\left(n_{2}, x\left(n_{2}\right)\right)\right) \leq b(\lambda) .
\end{aligned}
$$

Similarly, it can be deduced that

$$
V(n, x(n)) \leq \psi^{-1}(b(\lambda)), \quad n \in\left[n_{2}, n_{3}\right] \cap \mathbb{Z}_{+} .
$$

By simple induction, we can prove that

$$
\begin{aligned}
V(n, x(n)) \leq \psi^{-1} & (b(\lambda)), \quad n \in\left[n_{k}, n_{k+1}\right] \cap \mathbb{Z}_{+}, k \in \mathbb{Z}_{+}, \\
V\left(n_{k+1}, \bar{x}\left(n_{k+1}\right)\right) & =V\left(n_{k+1}, x\left(n_{k+1}\right)+I_{k+1}\left(n_{k+1}, x\left(n_{k+1}\right)\right)\right) \\
& \leq \psi\left(V\left(n_{k+1}, x\left(n_{k+1}\right)\right)\right) \\
& \leq b(\lambda) \\
& <\psi^{-1}(b(\lambda)) .
\end{aligned}
$$

It follows from conditions (ii) and (v) that

$$
\begin{gathered}
V(n, x(n)) \leq \psi^{-1}(b(\lambda))<a(A), \\
\|x(n)\| \leq a^{-1}(V(n, x(n))) \\
<a^{-1}(a(A))=A, \quad n \geq n_{0}, n \in \mathbb{Z}_{+} .
\end{gathered}
$$


This inequality implies that the system (1) with respect to $(\lambda, A)$ is uniformly practically stable.

Next, we show that the system (1) with respect to $(\lambda, A)$ is uniformly asymptotically practically stable. For any $\epsilon, 0<$ $\epsilon<A$, there exist numbers $a=a(\epsilon)>0,0<d<a$, such that

$$
\begin{gathered}
P(s)>\psi^{-1}(s)+a, \\
\psi^{-1}(s)+a>\psi^{-1}(s+d), \\
s \in\left[a(\epsilon), \psi^{-1}(b(\lambda))\right] .
\end{gathered}
$$

Let $N=N(\epsilon) \in \mathbb{Z}_{+}$satisfy $a(\epsilon)+(N-1) d \leq \psi^{-1}(b(\lambda)) \leq$ $a(\epsilon)+N d$, and $T=(N-1) v r, T \in \mathbb{Z}_{+}$, where $v \geq 1$. We will prove that

$$
V(n, x(n)) \leq \psi^{-1}(a(\epsilon)), \quad n \geq n_{0}+T, n \in \mathbb{Z}_{+} .
$$

In order to do this, we first prove that there exists a $T_{1} \geq n_{0}$, $T_{1} \in \mathbb{Z}_{+}$, such that

$$
V\left(T_{1}, x\left(T_{1}\right)\right) \leq a(\epsilon)+(N-1) d .
$$

If (25) does not hold, then, for any $n \geq n_{0}, n \in \mathbb{Z}_{+}$, $V(n, x(n))>a(\epsilon)+(N-1) d$.

Note that, for $s \in J$,

$$
\begin{aligned}
P(V(n, x(n))) & >\psi^{-1}(V(n, x(n)))+a \\
& \geq \psi^{-1}(a(\epsilon)+(N-1) d)+a \\
& >\psi^{-1}(a(\epsilon)+N d) \geq \psi^{-1}(b(\lambda)) \\
& \geq V(n+s, x(n+s)) .
\end{aligned}
$$

Thus,

$$
\Delta V(n, x(n)) \leq \omega(V(n, \bar{x}(n))), \quad n \geq n_{0} .
$$

Hence, we obtain

$$
\begin{aligned}
V\left(n_{2}, \bar{x}\left(n_{2}\right)\right)-V\left(n_{1}-1, \bar{x}\left(n_{1}-1\right)\right) \\
=V\left(n_{2}, \bar{x}\left(n_{2}\right)\right)-V\left(n_{2}, x\left(n_{2}\right)\right)+V\left(n_{2}, x\left(n_{2}\right)\right) \\
\quad-V\left(n_{2}-1, x\left(n_{2}-1\right)\right)+V\left(n_{2}-1, x\left(n_{2}-1\right)\right) \\
\quad-\cdots+V\left(n_{1}+1, x\left(n_{1}+1\right)\right)-V\left(n_{1}, \bar{x}\left(n_{1}\right)\right) \\
\quad+V\left(n_{1}, \bar{x}\left(n_{1}\right)\right)-V\left(n_{1}, x\left(n_{1}\right)\right) \\
\quad+V\left(n_{1}, x\left(n_{1}\right)\right)-V\left(n_{1}-1, x\left(n_{1}-1\right)\right)
\end{aligned}
$$

$$
\begin{aligned}
& \leq \psi\left(V\left(n_{2}, x\left(n_{2}\right)\right)\right)-V\left(n_{2}, x\left(n_{2}\right)\right) \\
& +\psi\left(V\left(n_{1}, x\left(n_{1}\right)\right)\right)-V\left(n_{1}, x\left(n_{1}\right)\right) \\
& +\Delta V\left(n_{2}-1, x\left(n_{2}-1\right)\right)+\Delta V\left(n_{2}-2, x\left(n_{2}-2\right)\right) \\
& +\cdots+\Delta V\left(n_{1}-1, x\left(n_{1}-1\right)\right) \\
& \leq \psi\left(V\left(n_{2}, x\left(n_{2}\right)\right)\right)-V\left(n_{2}, x\left(n_{2}\right)\right) \\
& +\psi\left(V\left(n_{1}, x\left(n_{1}\right)\right)\right)-V\left(n_{1}, x\left(n_{1}\right)\right) \\
& +\omega\left(V\left(n_{2}-1, x\left(n_{2}-1\right)\right)\right) \\
& +\omega\left(V\left(n_{2}-2, x\left(n_{2}-2\right)\right)\right) \\
& +\cdots+\omega\left(V\left(n_{1}-1, x\left(n_{1}-1\right)\right)\right) \\
& \leq V\left(n_{2}, x\left(n_{2}\right)\right)\left(\frac{\psi\left(V\left(n_{2}, x\left(n_{2}\right)\right)\right)}{V\left(n_{2}, x\left(n_{2}\right)\right)}-1\right) \\
& +V\left(n_{1}, x\left(n_{1}\right)\right)\left(\frac{\psi\left(V\left(n_{1}, x\left(n_{1}\right)\right)\right)}{V\left(n_{1}, x\left(n_{1}\right)\right)}-1\right) \\
& +V\left(n_{2}-1, x\left(n_{2}-1\right)\right) \frac{\omega\left(V\left(n_{2}-1, x\left(n_{2}-1\right)\right)\right)}{V\left(n_{2}-1, x\left(n_{2}-1\right)\right)} \\
& +V\left(n_{2}-2, x\left(n_{2}-2\right)\right) \frac{\omega\left(V\left(n_{2}-2, x\left(n_{2}-2\right)\right)\right)}{V\left(n_{2}-2, x\left(n_{2}-2\right)\right)} \\
& +\cdots+V\left(n_{1}-1, x\left(n_{1}-1\right)\right) \frac{\omega\left(V\left(n_{1}-1, x\left(n_{1}-1\right)\right)\right)}{V\left(n_{1}-1, x\left(n_{1}-1\right)\right)} \\
& \leq \psi^{-1}(b(\lambda))(p-1)+\psi^{-1}(b(\lambda)) q \tau \\
& +\psi^{-1}(b(\lambda))(p-1) \\
& \leq \psi^{-1}(b(\lambda))(2 p-2+q \tau) .
\end{aligned}
$$

Thus,

$$
V\left(n_{2}, \bar{x}\left(n_{2}\right)\right) \leq \psi^{-1}(b(\lambda))(2 p-1+q \tau)<0,
$$

which is a contradiction. Thus, there exists a $T_{1} \geq n_{0}, T_{1} \in \mathbb{Z}_{+}$, such that (25) holds.

Next, we prove that

$$
V(n, x(n)) \leq \psi^{-1}(a(\epsilon)+(N-1) d), \quad n \geq T_{1}, n \in \mathbb{Z}_{+} .
$$

Let $m=\min \left\{m \in \mathbb{Z}_{+}: n_{m} \geq T_{1}\right\}$, and we show that

$$
V(n, x(n)) \leq \psi^{-1}(a(\epsilon)+(N-1) d), \quad n \in\left[T_{1}, n_{m}\right] \cap \mathbb{Z}_{+} .
$$

If (31) does not hold, then there is a $r \in\left[T_{1}, n_{m}\right] \cap \mathbb{Z}_{+}$such that

$$
V(r, x(r))>\psi^{-1}(a(\epsilon)+(N-1) d) .
$$


Let $r^{\star}=\min \left\{n: V(n, x(n))>\psi^{-1}(a(\epsilon)+(N-1) d), n \epsilon\right.$ $\left.\left[T_{1}, t_{m}\right]\right\}$. Since

$$
V\left(T_{1}, x\left(T_{1}\right)\right) \leq a(\epsilon)+(N-1) d \leq \psi^{-1}(a(\epsilon)+(N-1) d),
$$

we have

$$
r^{\star}>T_{1}, \quad V\left(r^{\star}, x\left(r^{\star}\right)\right)>\psi^{-1}(a(\epsilon)+(N-1) d) .
$$

Let $\widehat{r}=\max \left\{n: V(n, x(n)) \leq a(\epsilon)+(N-1) d, n \in\left[T_{1}, r^{\star}\right)\right\}$. Note

$V\left(r^{\star}, x\left(r^{\star}\right)\right)>\psi^{-1}(a(\epsilon)+(N-1) d)>a(\epsilon)+(N-1) d$.

Thus,

$$
\begin{gathered}
\widehat{r}<r^{\star}, \quad V(\widehat{r}, x(\widehat{r})) \leq a(\epsilon)+(N-1) d, \\
a(\epsilon)+(N-1) d<V(n, x(n)) \leq \psi^{-1}(a(\epsilon)+(N-1) d), \\
n \in\left(\widehat{r}, r^{\star}\right) \cap \mathbb{Z}_{+} .
\end{gathered}
$$

Hence, we obtain

$$
\begin{aligned}
V\left(r^{\star},\right. & \left.x\left(r^{\star}\right)\right)-V(\widehat{r}, x(\widehat{r})) \\
& >\psi^{-1}(a(\epsilon)+(N-1) d)-(a(\epsilon)+(N-1) d) \\
& =\psi^{-1}(a(\epsilon)+(N-1) d)\left(1-\frac{a(\epsilon)+(N-1) d}{\psi^{-1}(a(\epsilon)+(N-1) d)}\right) \\
& \geq \psi^{-1}(a(\epsilon)+(N-1) d)(1-p) .
\end{aligned}
$$

On the other hand, note that, for any $n \in\left[\widehat{r}, r^{\star}\right] \cap \mathbb{Z}_{+}$,

$$
\begin{aligned}
P(V(n, x(n)))> & \psi^{-1}(V(n, x(n))) \\
& +a \geq \psi^{-1}(a(\epsilon)+(N-1) d)+a \\
> & \psi^{-1}(a(\epsilon)+N d) \geq \psi^{-1}(b(\lambda)) \\
\geq & V(n+s, x(n+s)), \quad s \in J .
\end{aligned}
$$

Using condition (iv), the inequality $\Delta V(n, x(n)) \leq \omega(V(n$, $\bar{x}(n)))=\omega(V(n, x(n)))$ holds for all $n \in\left[\widehat{r}, r^{\star}\right] \cap \mathbb{Z}_{+}$. Thus,

$$
\begin{aligned}
& V\left(r^{\star}, x\left(r^{\star}\right)\right)-V(\widehat{r}, x(\widehat{r})) \\
&= V\left(r^{\star}, x\left(r^{\star}\right)\right)-V\left(r^{\star}-1, x\left(r^{\star}-1\right)\right) \\
&+V\left(r^{\star}-1, x\left(r^{\star}-1\right)\right)-V\left(r^{\star}-2, x\left(r^{\star}-2\right)\right) \\
&+\cdots+V(\widehat{r}+1, x(\widehat{r}+1))-V(\widehat{r}, x(\widehat{r})) \\
&= \Delta V\left(r^{\star}-1, x\left(r^{\star}-1\right)\right)+\Delta V\left(r^{\star}-2, x\left(r^{\star}-2\right)\right) \\
&+\cdots+\Delta V(\widehat{r}, x(\widehat{r})) \\
& \leq \omega\left(V\left(r^{\star}-1, x\left(r^{\star}-1\right)\right)\right)+\omega\left(V\left(r^{\star}-2, x\left(r^{\star}-2\right)\right)\right) \\
&+\cdots+\omega(V(\widehat{r}, x(\widehat{r}))) \\
& \leq V\left(r^{\star}-1, x\left(r^{\star}-1\right)\right) \frac{\omega\left(V\left(r^{\star}-1, x\left(r^{\star}-1\right)\right)\right)}{V\left(r^{\star}-1, x\left(r^{\star}-1\right)\right)} \\
&+\cdots+V(\widehat{r}, x(\widehat{r})) \frac{\omega(V(\widehat{r}, x(\widehat{r})))}{V(\widehat{r}, x(\widehat{r}))} \\
& \leq \psi-1(a(\epsilon)+(N-1) d) q \tau .
\end{aligned}
$$

From (37) and (39), it can be deduced that $1-p<q \tau$, which is a contradiction. Thus (31) holds.

Then, from condition (iv), we get

$$
\begin{aligned}
V\left(n_{m}, \bar{x}\left(n_{m}\right)\right) & =V\left(n_{m}, x\left(n_{m}\right)+I_{m}\left(n_{m}, x\left(n_{m}\right)\right)\right) \\
& \leq \psi\left(V\left(n_{m}, x\left(n_{m}\right)\right)\right) \leq a(\epsilon)+(N-1) d .
\end{aligned}
$$

Similarly, it can be deduced that

$$
\begin{array}{r}
V(n, x(n)) \leq \psi^{-1}(a(\epsilon)+(N-1) d), \\
n \in\left[n_{m}, n_{m+1}\right] \cap \mathbb{Z}_{+} .
\end{array}
$$

By simple induction, one may derive that

$$
\begin{aligned}
& V(n, x(n)) \leq \psi^{-1}(a(\epsilon)+(N-1) d), \\
& n \in\left[n_{k}, n_{k+1}\right] \cap \mathbb{Z}_{+}, \quad k \geq m, \\
& V\left(n_{k+1}, \bar{x}\left(n_{k+1}\right)\right) \\
& \quad=V\left(n_{k+1}, x\left(n_{k+1}\right)+I_{k+1}\left(n_{k+1}, x\left(n_{k+1}\right)\right)\right) \\
& \quad \leq \psi\left(V\left(n_{k+1}, x\left(n_{k+1}\right)\right)\right) \\
& \quad \leq a(\epsilon)+(N-1) d .
\end{aligned}
$$

Thus, (30) holds.

Similarly, we can prove that there exists a $T_{2} \geq T_{1}+v r$, $v \geq 1$ such that

$$
V\left(T_{2}, x\left(T_{2}\right)\right) \leq a(\epsilon)+(N-2) d .
$$


By simple induction, we can prove, in general, that

$$
\begin{array}{r}
V\left(T_{j}, x\left(T_{j}\right)\right) \leq a(\epsilon)+(N-j) d, \\
V(n, x(n)) \leq \psi^{-1}(a(\epsilon)+(N-j) d), \\
n \geq T_{j}, \quad j=1,2, \ldots, N .
\end{array}
$$

Therefore, when choosing $j=N$, we obtain

$$
V(n, x(n)) \leq \psi^{-1}(a(\epsilon)), \quad n \geq T_{N},
$$

where $T_{N} \geq n_{0}+(N-1) v \tau$. Therefore,

$$
\|x\| \leq a^{-1}\left(\psi^{-1}(a(\epsilon))\right), \quad n \geq n_{0}+T
$$

where $T=(N-1) v r$. The proof is complete.

Remark 3. It can be found from Theorem 2 that it requires that the distance between two adjacent impulse times cannot be too long, and meanwhile the function $V$ should decrease at impulse times. We can see that impulses do contribute to the system's practical stability behavior. In the following, another result will be presented from the impulsive perturbation point of view, which is different from Theorem 2.

Theorem 4. Assume that there exist functions $a, b \in K, V$ : $\mathbb{Z}_{+} \times \mathbb{R}^{m} \rightarrow \mathbb{R}_{+}$such that

(i) $0<\lambda<A$ are given,

(ii) $a(\|x\|) \leq V(n, x) \leq b(\|x\|)$ for $(n, x) \in \mathbb{Z}_{+} \times \mathbb{R}^{m}$,

(iii) $V\left(n_{k}, \bar{x}\left(n_{k}\right)\right)=V\left(n_{k}, x\left(n_{k}\right)+I_{k}\left(n_{k}, x\left(n_{k}\right)\right)\right) \leq(1+$ $\left.\beta_{k}\right) V\left(n_{k}, x\left(n_{k}\right)\right)$, where $\beta_{k} \geq 0, \sum_{k=1}^{\infty} \beta_{k}<\infty$,

(iv) $V(n, x(n)) \geq V(n+s, x(n+s)), s \in J$, implies that

$\Delta V(n, x(n))=V(n, x(n))-V(n-1, \bar{x}(n-1)) \leq 0$,

where $x(n)$ is a solution of system (1),

(v) $M b(\lambda)<a(A), M=\prod_{k=1}^{\infty}\left(1+\beta_{k}\right)<\infty$.

Then, the system (1) with respect to $(\lambda, A)$ is uniformly practically stable.

Proof. For any $n_{0} \geq 0$, let $\bar{x}(n) \doteq \bar{x}\left(n, n_{0}, \varphi\right)$ be the solution of system (1) through $\left(n_{0}, \varphi\right)$, where $\left(n_{0}, \varphi\right) \in \mathbb{Z}_{+} \times S$ and $\|\varphi\|_{J}<$ $\lambda$. It suffices to show that

$$
\|x\|<A, \quad n \geq n_{0}, \quad n \in \mathbb{Z}_{+} .
$$

Next, we prove that

$$
V(n, x(n)) \leq M b(\lambda), \quad n \in\left[n_{0},+\infty\right) \cap \mathbb{Z}_{+} .
$$

First, we show that

$$
V(n, x(n)) \leq b(\lambda), \quad n \in\left[n_{0}, n_{1}\right] \cap \mathbb{Z}_{+} .
$$

If it does not hold, then there exists a $r \in\left[n_{0}, n_{1}\right] \cap \mathbb{Z}_{+}$such that

$$
V(r, x(r))>b(\lambda)
$$

Let $r_{1}=\min \left\{n: V(n, x(n))>b(\lambda), n \in\left[n_{0}, n_{1}\right] \cap \mathbb{Z}_{+}\right\}$. Since $V\left(n_{0}, x\left(n_{0}\right)\right) \leq b(\lambda)$, it is clear that

$$
\begin{gathered}
V\left(r_{1}, x\left(r_{1}\right)\right)>b(\lambda), \\
V\left(r_{1}-1, x\left(r_{1}-1\right)\right) \leq b(\lambda), \\
\Delta V\left(r_{1}, x\left(r_{1}\right)\right)=V\left(r_{1}, x\left(r_{1}\right)\right)-V\left(r_{1}-1, \bar{x}\left(r_{1}-1\right)\right)>0, \\
r_{1}>n_{0} .
\end{gathered}
$$

Thus, for $s \in J$,

$$
V\left(r_{1}, x\left(r_{1}\right)\right)>b(\lambda) \geq V\left(r_{1}+s, x\left(r_{1}+s\right)\right) .
$$

By condition (iv), we have that

$$
\Delta V\left(r_{1}, x\left(r_{1}\right)\right) \leq 0
$$

which is a contradiction. Thus, (50) holds.

From (50) and condition (iii), we obtain

$$
V\left(n_{1}, \bar{x}\left(n_{1}\right)\right) \leq\left(1+\beta_{1}\right) V\left(n_{1}, x\left(n_{1}\right)\right) \leq\left(1+\beta_{1}\right) b(\lambda) .
$$

Next, we show that

$$
V(n, x(n)) \leq\left(1+\beta_{1}\right) b(\lambda), \quad n \in\left[n_{1}, n_{2}\right] \cap \mathbb{Z}_{+} .
$$

If this assertion is not true, then there exists a $r \in\left[n_{1}, n_{2}\right] \cap \mathbb{Z}_{+}$ such that

$$
V(r, x(r))>\left(1+\beta_{1}\right) b(\lambda) .
$$

Let $r_{2}=\min \left\{n: V(n, x(n))>\left(1+\beta_{1}\right) b(\lambda), n \in\left[n_{1}, n_{2}\right] \cap \mathbb{Z}_{+}\right\}$. Since $V\left(n_{1}, x\left(n_{1}\right)\right) \leq\left(1+\beta_{1}\right) b(\lambda)$, we get

$$
\begin{gathered}
V\left(r_{2}, x\left(r_{2}\right)\right)>\left(1+\beta_{1}\right) b(\lambda), \\
V\left(r_{2}-1, x\left(r_{2}-1\right)\right) \leq\left(1+\beta_{1}\right) b(\lambda), \\
\Delta V\left(r_{2}, x\left(r_{2}\right)\right)=V\left(r_{2}, x\left(r_{2}\right)\right)-V\left(r_{2}-1, \bar{x}\left(r_{2}-1\right)\right)>0, \\
r_{2}>n_{1} .
\end{gathered}
$$

Thus, for $s \in J$,

$$
V\left(r_{2}, x\left(r_{2}\right)\right)>\left(1+\beta_{2}\right) b(\lambda) \geq V\left(r_{2}+s, x\left(r_{2}+s\right)\right) .
$$

By condition (iv), we have

$$
\Delta V\left(r_{2}, x\left(r_{2}\right)\right) \leq 0
$$

which is a contradiction. Thus, (56) holds.

Considering (30) and condition (iii), it can be deduced that

$$
\begin{aligned}
V\left(n_{2}, \bar{x}\left(n_{2}\right)\right) & \leq\left(1+\beta_{2}\right) V\left(n_{2}, x\left(n_{2}\right)\right) \\
& \leq\left(1+\beta_{1}\right)\left(1+\beta_{2}\right) b(\lambda) .
\end{aligned}
$$


By simple induction, we have

$$
\begin{array}{r}
V(n, x(n)) \leq\left(1+\beta_{1}\right)\left(1+\beta_{2}\right) \cdots\left(1+\beta_{k}\right) b(\lambda), \\
n \in\left[n_{k}, n_{k+1}\right] \cap \mathbb{Z}_{+}, \quad k \in \mathbb{Z}_{+},
\end{array}
$$

which, together with (50) and condition (v), yields that

$$
V(n, x(n)) \leq M b(\lambda)<a(A), \quad n \geq n_{0}, n \in \mathbb{Z}_{+} .
$$

Therefore, from condition (ii), we have

$$
\|x\| \leq A, \quad n \geq n_{0}, \quad n \in \mathbb{Z}_{+} .
$$

Thus, system (1) with respect to $(\lambda, A)$ is uniformly practically stable.

The proof is complete.

Theorem 5. Assume that there exist functions $a, b \in K, P, \omega \in$ $K_{1}, V: \mathbb{Z}_{+} \times \mathbb{R}^{m} \rightarrow \mathbb{R}_{+}$, such that

(i) $0<\lambda<A$ are given,

(ii) $a(\|x\|) \leq V(n, x) \leq b(\|x\|)$ for $(n, x) \in \mathbb{Z}_{+} \times \mathbb{R}^{m}$,

(iii) $V\left(n_{k}, \bar{x}\left(n_{k}\right)\right)=V\left(n_{k}, x\left(n_{k}\right)+I_{k}\left(n_{k}, x\left(n_{k}\right)\right)\right) \leq(1+$ $\left.\beta_{k}\right) V\left(n_{k}, x\left(n_{k}\right)\right)$, where $\beta_{k} \geq 0, \sum_{k=1}^{\infty} \beta_{k}<\infty$,

(iv) $P(V(n, x(n))) \geq V(n+s, x(n+s)), s \in J$, implies that

$$
\begin{aligned}
\Delta V(n, x(n)) & =V(n, x(n))-V(n-1, \bar{x}(n-1)) \\
& \leq-\omega(V(n, x(n))),
\end{aligned}
$$

with $P(s)>M s, s>0, M=\prod_{k=1}^{\infty}\left(1+\beta_{k}\right)<\infty$, where $x(n)$ is a solution of system (1);

(v) $M b(\lambda)<a(A)$.

Then, the system (1) with respect to $(\lambda, A)$ is uniformly asymptotically practically stable.

Proof. For any $n_{0} \geq 0$, let $\bar{x}(n) \doteq \bar{x}\left(n, n_{0}, \varphi\right)$ be the solution of system (1) through $\left(n_{0}, \varphi\right)$, where $\left(n_{0}, \varphi\right) \in \mathbb{Z}_{+} \times S$, and $\|\varphi\|_{J}<\lambda$. From Theorem 4 , it is easy to see that the system (1) with respect to $(\lambda, A)$ is uniformly practically stable. Now, we show that the system (1) with respect to $(\lambda, A)$ is uniformly asymptotically practically stable.

For any $\epsilon \in(0, A)$, there exists number $d=d(\epsilon)>0$ such that

$$
P(s)>M s+d, \quad s \in\left[\frac{a(\epsilon)}{M}, M b(\lambda)\right] .
$$

Let $N=N(\epsilon)$ be the smallest positive integer such that

$$
\begin{gathered}
\frac{a(\epsilon)+N d}{M} \geq M b(\lambda), \\
\gamma=\inf _{a(\epsilon) / M \leq s \leq M b(\lambda)} \omega(s)>(1+M \bar{M}) b(\lambda), \quad \bar{M}=\sum_{k=1}^{\infty} \beta_{k} .
\end{gathered}
$$

We will prove that there exists $T \in \mathbb{Z}_{+}$such that

$$
V(n, x(n)) \leq a(\epsilon), \quad n \geq n_{0}+T, n \in \mathbb{Z}_{+} .
$$

To this end, we first prove that there exists $T_{1} \in\left(n_{k}, n_{k+1}\right) \cap \mathbb{Z}_{+}$, $k \in \mathbb{Z}_{+}$, such that

$$
V(n, x(n)) \leq a(\epsilon)+(N-1) d, \quad n \geq T_{1}, n \in \mathbb{Z}_{+} .
$$

In fact, when $n \in\left[n_{0}, T_{1}\right]$, there exists a $N_{1} \in\left[n_{m}, n_{m+1}\right) \cap$ $\mathbb{Z}_{+} \subseteq\left[n_{0}, T_{1}\right] \cap \mathbb{Z}_{+}, m \in \mathbb{Z}_{+}$, such that

$$
V\left(N_{1}, x\left(N_{1}\right)\right) \leq \frac{a(\epsilon)+(N-1) d}{M} .
$$

If (70) does not hold, it is clear that, for any $n \in\left[n_{0}, T_{1}\right] \cap \mathbb{Z}_{+}$,

$$
\begin{aligned}
& V(n, x(n))>\frac{a(\epsilon)+(N-1) d}{M}, \\
& \frac{a(\epsilon)}{M} \leq V(n, x(n)) \leq M b(\lambda) .
\end{aligned}
$$

Thus, for $s \in J$,

$$
\begin{aligned}
P(V(n, x(n))) \geq & M V(n, x(n))+d \geq a(\epsilon) \\
& +(N-1) d+d=a(\epsilon)+N d \\
\geq & M b(\lambda) \geq V(n+s, x(n+s)) .
\end{aligned}
$$

It follows from condition (iv) that

$$
\Delta V(n, x(n)) \leq-\omega(V(n, x(n))) \leq-\gamma .
$$

On the other hand, since there at least exists one point $T_{1}$ which is not an impulsive point, we obtain

$$
\begin{aligned}
V\left(T_{1}, x\left(T_{1}\right)\right)-V\left(n_{0}, x\left(n_{0}\right)\right) \\
=V\left(T_{1}, x\left(T_{1}\right)\right)-V\left(T_{1}-1, x\left(T_{1}-1\right)\right) \\
\quad+V\left(T_{1}-1, x\left(T_{1}-1\right)\right)-V\left(T_{1}-2, x\left(T_{1}-2\right)\right) \\
\quad+\cdots+V\left(n_{0}+1, x\left(n_{0}+1\right)\right)-V\left(n_{0}, x\left(n_{0}\right)\right) \\
=\Delta V\left(T_{1}, x\left(T_{1}\right)\right)+\Delta V\left(T_{1}-1, x\left(T_{1}-1\right)\right) \\
\quad+\cdots+\Delta V\left(n_{0}+1, x\left(n_{0}+1\right)\right) \\
\quad-\omega\left(V\left(T_{1}, x\left(T_{1}\right)\right)\right)+\sum_{j=1}^{k} V\left(n_{j-1}, x\left(n_{j-1}\right)\right) \beta_{j} \\
\leq \\
\leq
\end{aligned}
$$

Thus,

$$
V\left(T_{1}, x\left(T_{1}\right)\right) \leq M b(\lambda)(1+\bar{M})-\gamma<0,
$$

which is a contradiction. Hence, when $n \in\left[n_{0}, T_{1}\right] \cap \mathbb{Z}_{+}$, there exists a $N_{1} \in\left[n_{m}, n_{m+1}\right) \cap \mathbb{Z}_{+} \subseteq\left[n_{0}, T_{1}\right] \cap \mathbb{Z}_{+}, m \in \mathbb{Z}_{+}$, such that

$$
V\left(N_{1}, x\left(N_{1}\right)\right) \leq \frac{a(\epsilon)+(N-1) d}{M} .
$$

Then, we claim that

$$
V(n, x(n)) \leq \frac{a(\epsilon)+(N-1) d}{M}, \quad n \in\left[N_{1}, n_{m+1}\right] \cap \mathbb{Z}_{+} .
$$


If (77) does not hold, there exists a $\widehat{N} \in\left[N_{1}, n_{m+1}\right] \cap \mathbb{Z}_{+}$such that

$$
V(\widehat{N}, x(\widehat{N}))>\frac{a(\epsilon)+(N-1) d}{M} .
$$

Let $\widetilde{N}=\min \{n: V(n, x(n))>(a(\epsilon)+(N-1) d) / M, n \epsilon$ $\left.\left[N_{1}, n_{m+1}\right] \cap \mathbb{Z}_{+}\right\}$. Since

$$
V\left(N_{1}, x\left(N_{1}\right)\right) \leq \frac{a(\epsilon)+(N-1) d}{M},
$$

we have that

$$
\begin{gathered}
V(\widetilde{N}, x(\widetilde{N}))>\frac{a(\epsilon)+(N-1) d}{M}, \\
\Delta V(\widetilde{N}, x(\widetilde{N}))>0, \\
\widetilde{N}>N_{1} .
\end{gathered}
$$

Note that $a(\epsilon) / M \leq V(\widetilde{N}, x(\widetilde{N})) \leq M b(\lambda)$; thus for $s \in J$,

$$
\begin{aligned}
P(V(\widetilde{N}, x(\widetilde{N}))) \geq & M V(\widetilde{N}, x(\widetilde{N}))+d \geq a(\epsilon) \\
& +(N-1) d+d=a(\epsilon)+N d \\
\geq & M b(\lambda) \geq V(\widetilde{N}+s, x(\widetilde{N}+s)) .
\end{aligned}
$$

It follows from condition (iv) that

$$
\Delta V(\widetilde{N}, x(\widetilde{N})) \leq-\omega(V(\widetilde{N}, x(\widetilde{N})))<0,
$$

which is a contradiction. Thus, (77) holds.

Considering (77) and condition (iii), it can be deduced that

$$
\begin{aligned}
V\left(n_{m+1}, \bar{x}\left(n_{m+1}\right)\right) & \leq\left(1+\beta_{m+1}\right) V\left(n_{m+1}, x\left(n_{m+1}\right)\right) \\
& \leq\left(1+\beta_{m+1}\right) \frac{a(\epsilon)+(N-1) d}{M} .
\end{aligned}
$$

Similarly, we may show

$$
\begin{gathered}
V(n, x(n)) \\
\leq\left(1+\beta_{m+1}\right) \frac{a(\epsilon)+(N-1) d}{M}, \\
n \in\left[n_{m+1}, n_{m+2}\right], \\
V\left(n_{m+2}, \bar{x}\left(n_{m+2}\right)\right) \leq\left(1+\beta_{m+2}\right) V\left(n_{m+2}, x\left(n_{m+2}\right)\right) \\
\leq\left(1+\beta_{m+2}\right)\left(1+\beta_{m+1}\right) \\
\times \frac{a(\epsilon)+(N-1) d}{M} .
\end{gathered}
$$

By simple induction, we can prove in general that

$$
\begin{aligned}
& V(n, x(n)) \\
& \leq \prod_{j=1}^{i}\left(1+\beta_{m+j}\right) \frac{a(\epsilon)+(N-1) d}{M} \\
& \leq a(\epsilon)+(N-1) d, \quad n \in\left[n_{m+i}, n_{m+i+1}\right], i \in \mathbb{Z}_{+} .
\end{aligned}
$$

Thus, (69) holds.
Next, we prove that there exists $T_{2} \in\left(n_{l}, n_{l+1}\right) \cap \mathbb{Z}_{+}, l \in \mathbb{Z}_{+}$, $T_{2}>T_{1}+q r, q>1, q \in \mathbb{Z}_{+}$, such that

$$
V(n, x(n)) \leq a(\epsilon)+(N-2) d, \quad n \geq T_{2}, n \in \mathbb{Z}_{+} .
$$

In fact, when $n \in\left[T_{1}+r, T_{2}\right]$, there exists a $N_{2} \in\left[n_{\bar{m}}, n_{\bar{m}+1}\right] \cap$ $\mathbb{Z}_{+} \subseteq\left[T_{1}+r, T_{2}\right] \cap \mathbb{Z}_{+}, \bar{m} \in \mathbb{Z}_{+}$, such that

$$
V\left(N_{2}, x\left(N_{2}\right)\right) \leq \frac{a(\epsilon)+(N-1) d}{M} .
$$

If (87) does not hold, it is clear that, for any $n \in\left[T_{1}+r, T_{2}\right] \cap$ $\mathbb{Z}_{+}$,

$$
\begin{aligned}
& V(n, x(n))>\frac{a(\epsilon)+(N-2) d}{M}, \\
& \frac{a(\epsilon)}{M} \leq V(n, x(n)) \leq M b(\lambda) .
\end{aligned}
$$

Thus, for $s \in J$,

$$
\begin{aligned}
P(V(n, x(n))) \geq & M V(n, x(n))+d \geq a(\epsilon) \\
& +(N-2) d+d=a(\epsilon)+(N-1) d \\
\geq & M b(\lambda) \geq V(n+s, x(n+s)) .
\end{aligned}
$$

It follows from condition (iv) that

$$
\Delta V(n, x(n)) \leq-\omega(V(n, x(n))) \leq-\gamma .
$$

On the other hand, since there at least exists one point $T_{2}$ which is not an impulsive point, we obtain

$$
\begin{aligned}
V\left(T_{2}, x\left(T_{2}\right)\right)-V\left(T_{1}+r, x\left(T_{1}+r\right)\right) \\
=V\left(T_{2}, x\left(T_{2}\right)\right)-V\left(T_{2}-1, x\left(T_{2}-1\right)\right) \\
\quad+V\left(T_{2}-1, x\left(T_{2}-1\right)\right)-V\left(T_{2}-2, x\left(T_{2}-2\right)\right) \\
\quad+\cdots+V\left(T_{1}+r+1, x\left(T_{1}+r+1\right)\right) \\
\quad-V\left(T_{1}+r, x\left(T_{1}+r\right)\right) \\
=\Delta V\left(T_{2}, x\left(T_{2}\right)\right)+\Delta V\left(T_{2}-1, x\left(T_{2}-1\right)\right) \\
\quad+\cdots+\Delta V\left(T_{1}+r+1, x\left(T_{1}+r+1\right)\right) \\
\leq-\omega\left(V\left(T_{2}, x\left(T_{2}\right)\right)\right)+\sum_{j=T_{1}+r}^{n_{\bar{m}}} V\left(n_{j-1}, x\left(n_{j-1}\right)\right) \beta_{j} \\
\leq-\gamma+M \bar{M} b(\lambda) .
\end{aligned}
$$

Thus,

$$
V\left(T_{2}, x\left(T_{2}\right)\right) \leq M b(\lambda)(1+\bar{M})-\gamma \leq 0,
$$

which is a contradiction. Hence, when $n \in\left[T_{1}+r, T_{2}\right] \cap \mathbb{Z}_{+}$, there exists a $N_{2} \in\left[n_{\bar{m}}, n_{\bar{m}+1}\right) \subseteq\left[T_{1}+r, T_{2}\right] \cap \mathbb{Z}_{+}, \bar{m} \in \mathbb{Z}_{+}$, such that

$$
V\left(N_{2}, x\left(N_{2}\right)\right) \leq \frac{a(\epsilon)+(N-2) d}{M} .
$$

Similarly, we can prove that (86) holds. 
By simple induction, we have that

$$
\begin{gathered}
V(n, x(n)) \leq a(\epsilon)+(N-i) d, \\
n \geq n_{0}+T_{i}, \quad i=1,2, \ldots, N, \quad n \in \mathbb{Z}_{+} .
\end{gathered}
$$

Therefore, when choosing $i=N$, we obtain

$$
V(n, x(n)) \leq a(\epsilon), \quad n \geq n_{0}+T_{N}, n \in \mathbb{Z}_{+} .
$$

From condition (ii), we have that

$$
\|x(n)\| \leq \epsilon, \quad n \geq n_{0}+T_{N}, n \in \mathbb{Z}_{+} .
$$

The proof is complete.

\section{Applications}

The following illustrative examples will demonstrate the effectiveness of our results.

Example 6. Consider the following impulsive discrete system:

$$
\begin{aligned}
& x(n+1)=c x(n)+d x(n-1), \quad n \geq n_{0}, n \in \mathbb{Z}_{+}, \\
& \bar{x}(n)= \begin{cases}x(n), & n \neq n_{k}, \quad k \in \mathbb{Z}_{+}, \\
\eta x\left(n_{k}\right), & n=n_{k}, k \in \mathbb{Z}_{+},\end{cases} \\
& x_{n_{0}}(s)=\varphi(s), \quad s \in J,
\end{aligned}
$$

where $c, d, \eta$ are any three constants and $0<\eta<1$.

Property 1 . Given constants $\lambda, A$ satisfy $\lambda<\eta A$, and there is a constant $\zeta>1 / \eta$.

Then, the system (97) with respect to $(\lambda, A)$ is uniformly asymptotically practically stable if

$$
\tau(|c-1|+\zeta|d|)<1-2 \eta,
$$

where $\tau \triangleq \max _{k \in \mathbb{Z}_{+}}\left\{n_{k+1}-n_{k}\right\}$.

Proof. Choose $V(n, x(n))=|x(n)|$, where $x(n)$ is a solution of system (97). Let $b(s)=a(s)=s, P(s)=\zeta s, \omega(s)=(|c-1|+$ $\zeta|d|) s, \psi(s)=\eta s, s>0$, and then

$$
\begin{aligned}
\Delta V & (n, x(n)) \\
& =V(n+1, x(n+1))-V(n, \bar{x}(n)) \\
& =|x(n+1)|-|x(n)| \\
& =|c x(n)+d x(n-1)|-|x(n)| \\
& \leq|(c-1) x(n)+d x(n-1)| \\
& \leq|c-1| \cdot|x(n)|+|d| \cdot|x(n-1)| \\
& =|c-1| V(n, x(n))+|d| V(n-1, x(n-1)) \\
& \leq|c-1| V(n, x(n))+|d| \zeta V(n, x(n)) \\
& =(|c-1|+\zeta|d|) V(n, x(n)) .
\end{aligned}
$$

By Theorem 2, the above property can be easily derived.
Example 7. Consider the following impulsive discrete system:

$$
\begin{aligned}
& x(n+1)=\frac{x(n)[(1+d) x(n-1)+c d]}{x(n-1)+c}, \quad n \geq n_{0}, n \in \mathbb{Z}_{+}, \\
& \bar{x}(n)= \begin{cases}x(n), & n \neq n_{k}, \quad k \in \mathbb{Z}_{+}, \\
\eta x\left(n_{k}\right), & n=n_{k}, k \in \mathbb{Z}_{+},\end{cases} \\
& x_{n_{0}}(s)=\varphi(s), \quad s \in J \text {, }
\end{aligned}
$$

where $c, d, \eta$ are three constants and $c>0,0<\eta<1$.

Property 2. Given constants $\lambda, A$ satisfy $\lambda<\eta A$, and there is a constant $\zeta>1 / \eta$.

Then, the system (100) with respect to $(\lambda, A)$ is uniformly asymptotically practically stable if

$$
\tau|d|<1-2 \eta
$$

where $\tau \triangleq \max _{k \in \mathbb{Z}_{+}}\left\{n_{k+1}-n_{k}\right\}$.

Proof. Choose $V(n, x(n))=|x(n)|$, where $x(n)$ is a solution of system (100). Let $b(s)=a(s)=s, P(s)=\zeta s, \omega(s)=s \cdot(|d|+$ $1 /(1+c / \zeta s)-1), \psi(s)=\eta s, s>0$, and then

$$
\begin{aligned}
\Delta V(n, & x(n)) \\
& =V(n+1, x(n+1))-V(n, \bar{x}(n)) \\
& =|x(n+1)|-|x(n)| \\
& =\left|\frac{x(n)[(1+d) x(n-1)+c d]}{x(n-1)+c}\right|-|x(n)| \\
& =|x(n)| \cdot\left(\left|\frac{d x(n-1)+c d+x(n-1)}{x(n-1)+c}\right|-1\right) \\
& =|x(n)| \cdot\left(\left|d+\frac{1}{1+c / x(n-1)}\right|-1\right) \\
& \leq|x(n)| \cdot\left(|d|+\frac{1}{1+c / V(n-1, x(n-1))}-1\right) \\
& \leq V(n, x(n)) \cdot\left(|d|+\frac{1}{1+c / \zeta V(n, x(n))}-1\right) \\
& =\omega(V(n)) .
\end{aligned}
$$

By Theorem 2, the above property can be easily derived.

Remark 8. According to Theorem 2, we obtained the sufficient conditions guaranteeing uniformly asymptotically practical stability of the two impulsive discrete systems, respectively. In other words, the results we presented are effective for both linear and nonlinear impulsive discrete systems.

\section{Conclusion}

In this paper, we considered the impulsive discrete systems with time delays. Based on Lyapunov functions and the 
Razumikhin-type technique, the practical stability and uniformly asymptotically practical stability have been presented, which is dependent on both the impulses and the time delays. To our knowledge, there is almost no result concerning the problem of practical stability for impulsive discrete systems with delays. According to the analysis, we can see that impulses do contribute to the system's practical stability behavior. Two examples have been illustrated to demonstrate the usefulness of the proposed method.

\section{Conflict of Interests}

The authors declare that there is no conflict of interests regarding the publication of this paper.

\section{References}

[1] Z. Zhang and X. Liu, "Robust stability of uncertain discrete impulsive switching systems," Computers \& Mathematics with Applications, vol. 58, no. 2, pp. 380-389, 2009.

[2] Q. Song and J. Cao, "Dynamical behaviors of discrete-time fuzzy cellular neural networks with variable delays and impulses," Journal of the Franklin Institute, vol. 345, no. 1, pp. 39-59, 2008.

[3] R. Nigmatulin and M. Kipnis, "Stability of the discrete population model with two delays," in Proceedings of the International Conference on Physics and Control, vol. 1, pp. 313-315, IEEE, 2003.

[4] M. Bohner and A. Peterson, Dynamic Equations on Time Scales: An Introduction with Applications, Birkhäuser, Boston, Mass, USA, 2001.

[5] Y. Zhang, J. Sun, and G. Feng, "Impulsive control of discrete systems with time delay," IEEE Transactions on Automatic Control, vol. 54, no. 4, pp. 871-875, 2009.

[6] B. Liu and H. J. Marquez, "Razumikhin-type stability theorems for discrete delay systems," Automatica, vol. 43, no. 7, pp. 12191225, 2007.

[7] X. Liu and Z. Zhang, "Uniform asymptotic stability of impulsive discrete systems with time delay," Nonlinear Analysis: Theory, Methods \& Applications, vol. 74, no. 15, pp. 4941-4950, 2011.

[8] Y. Zhang, "Exponential stability of impulsive discrete systems with time delays," Applied Mathematics Letters, vol. 25, no. 12, pp. 2290-2297, 2012.

[9] V. Lakshmikantham, S. Leela, and A. A. Martynyuk, Practical Stability of Nonlinear Systems, World Scientific, Singapore, 1990.

[10] C. H. Kou and S. N. Zhang, "Practical stability for finite delay differential systems in terms of two measures," Acta Mathematicae Applicatae Sinica, vol. 25, no. 3, pp. 476-483, 2002.

[11] D. D. Bainov and I. M. Stamova, "On the practical stability of the solutions of impulsive systems of differential-difference equations with variable impulsive perturbations," Journal of Mathematical Analysis and Applications, vol. 200, no. 2, pp. 272288, 1996.

[12] Y. Zhang and J. Sun, "Practical stability of impulsive functional differential equations in terms of two measurements," Computers \& Mathematics with Applications, vol. 48, no. 10-11, pp. 15491556, 2004.

[13] Y. Zhang and J. Sun, "Eventual practical stability of impulsive differential equations with time delay in terms of two measurements," Journal of Computational and Applied Mathematics, vol. 176, no. 1, pp. 223-229, 2005.
[14] R. Villafuerte, S. Mondié, and A. Poznyak, "Practical stability of time-delay systems: LMI's approach," European Journal of Control, vol. 17, no. 2, pp. 127-138, 2011.

[15] I. M. Stamova, "Vector Lyapunov functions for practical stability of nonlinear impulsive functional differential equations," Journal of Mathematical Analysis and Applications, vol. 325, no. 1, pp. 612-623, 2007.

[16] D. D. Baĭnov and P. S. Simeonov, Systems with Impulse Effect: Stability, Theory and Applications, Halsted Press, New York, NY, USA, 1989.

[17] V. Lakshmikantham, D. D. Bainnov, and P. S. Simeonov, Theory of Impulsive Differential Equations, vol. 6 of Series in Modern Applied Mathematics, World Scientific, Singapore, 1989.

[18] T. Yang, Impulsive Systems and Control: Theory and Applications, Nova Science Publishers, Huntington, NY, USA, 2001.

[19] X. Fu, B. Yan, and Y. Liu, Introduction of Impulsive Differential Systems, Science Press, Beijing, China, 2005.

[20] V. Lakshmikantham, V. M. Matrosov, and S. Sivasundaram, Vector Lyapunov Functions and Stability Analysis of Nonlinear Systems, vol. 63 of Mathematics and Its Applications, Kluwer Academic, Dordrecht, The Netherlands, 1991.

[21] J. H. Shen, "Razumikhin techniques in impulsive functionaldifferential equations," Nonlinear Analysis: Theory, Methods \& Applications, vol. 36, no. 1, pp. 119-130, 1999.

[22] Z. Luo and J. Shen, "New Razumikhin type theorems for impulsive functional differential equations," Applied Mathematics and Computation, vol. 125, no. 2-3, pp. 375-386, 2002.

[23] X. Li, "Further analysis on uniform stability of impulsive infinite delay differential equations," Applied Mathematics Letters, vol. 25, no. 2, pp. 133-137, 2012.

[24] X. Fu and X. Li, "Razumikhin-type theorems on exponential stability of impulsive infinite delay differential systems," Journal of Computational and Applied Mathematics, vol. 224, no. 1, pp. $1-10,2009$.

[25] X. Li, "New results on global exponential stabilization of impulsive functional differential equations with infinite delays or finite delays," Nonlinear Analysis: Real World Applications, vol. 11, no. 5, pp. 4194-4201, 2010.

[26] Z. Luo and J. Shen, "Stability of impulsive functional differential equations via the Liapunov functional," Applied Mathematics Letters, vol. 22, no. 2, pp. 163-169, 2009.

[27] X. Liu and Q. Wang, "The method of Lyapunov functionals and exponential stability of impulsive systems with time delay," Nonlinear Analysis: Theory, Methods \& Applications, vol. 66, no. 7, pp. 1465-1484, 2007.

[28] X. Li and M. Bohner, "An impulsive delay differential inequality and applications," Computers \& Mathematics with Applications, vol. 64, no. 6, pp. 1875-1881, 2012.

[29] X. Li, "Uniform asymptotic stability and global stabiliy of impulsive infinite delay differential equations," Nonlinear Analysis: Theory, Methods \& Applications, vol. 70, no. 5, pp. 1975-1983, 2009.

[30] X. Li, H. Akca, and X. Fu, "Uniform stability of impulsive infinite delay differential equations with applications to systems with integral impulsive conditions," Applied Mathematics and Computation, vol. 219, no. 14, pp. 7329-7337, 2013. 


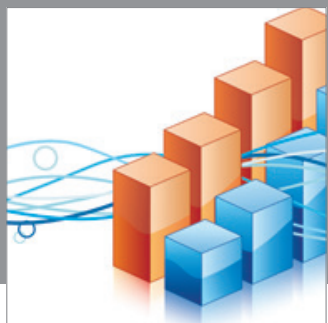

Advances in

Operations Research

mansans

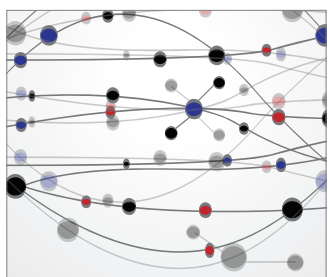

The Scientific World Journal
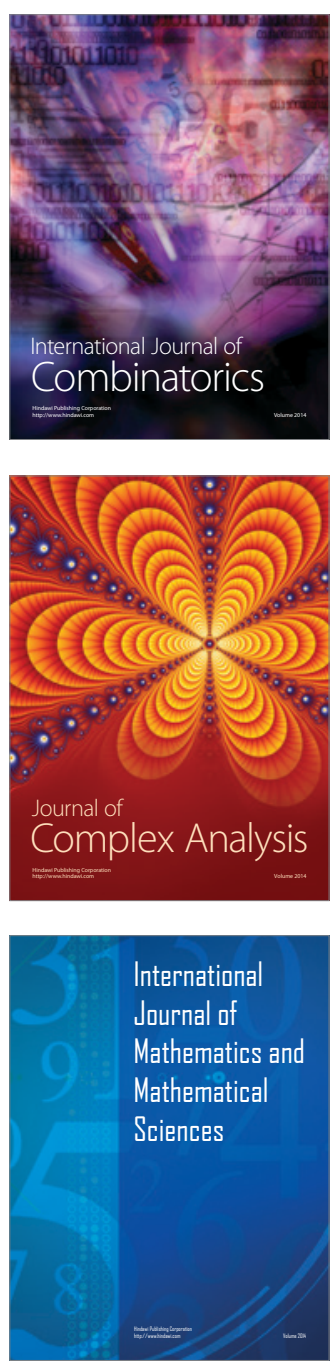
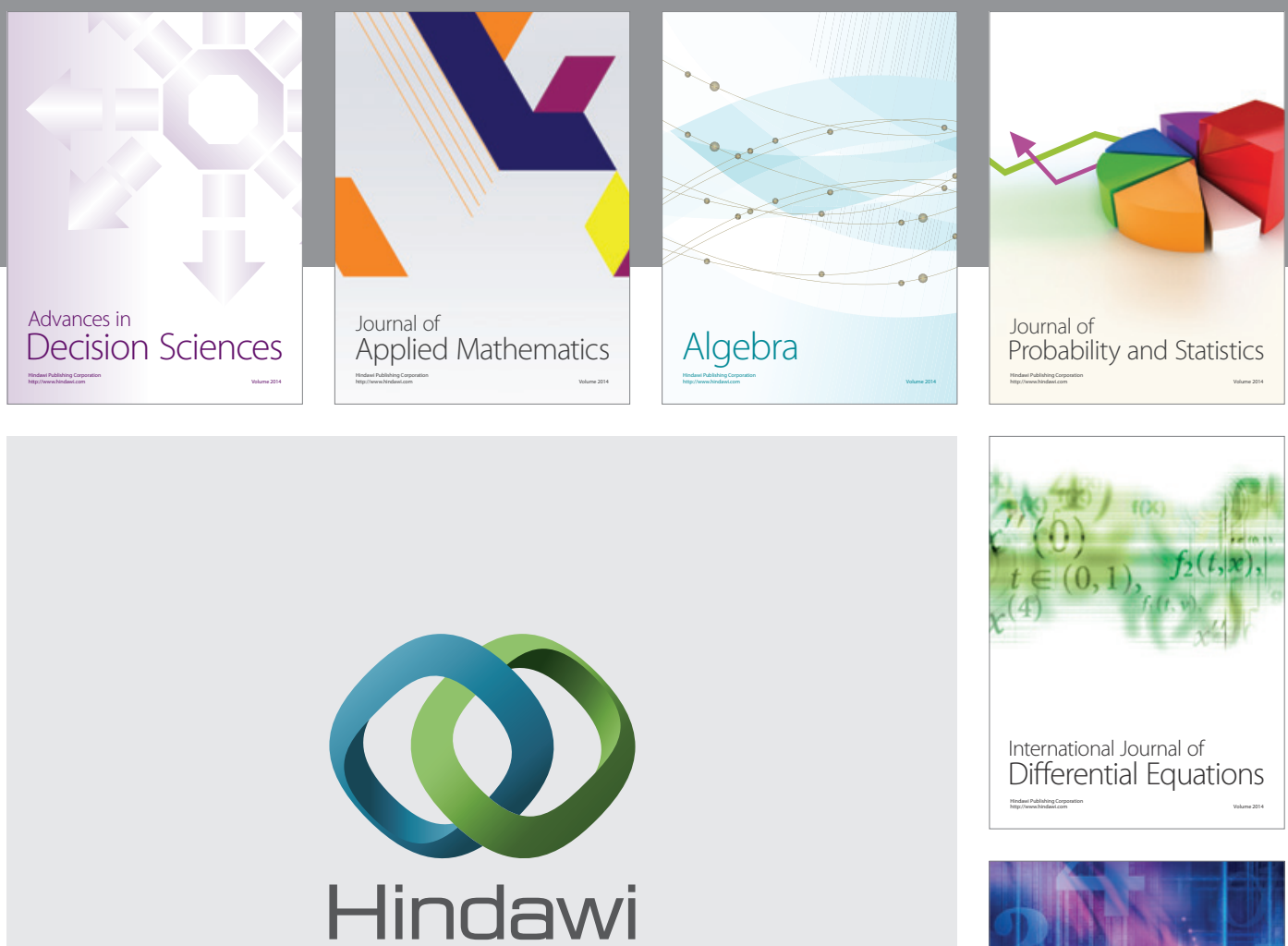

Submit your manuscripts at http://www.hindawi.com
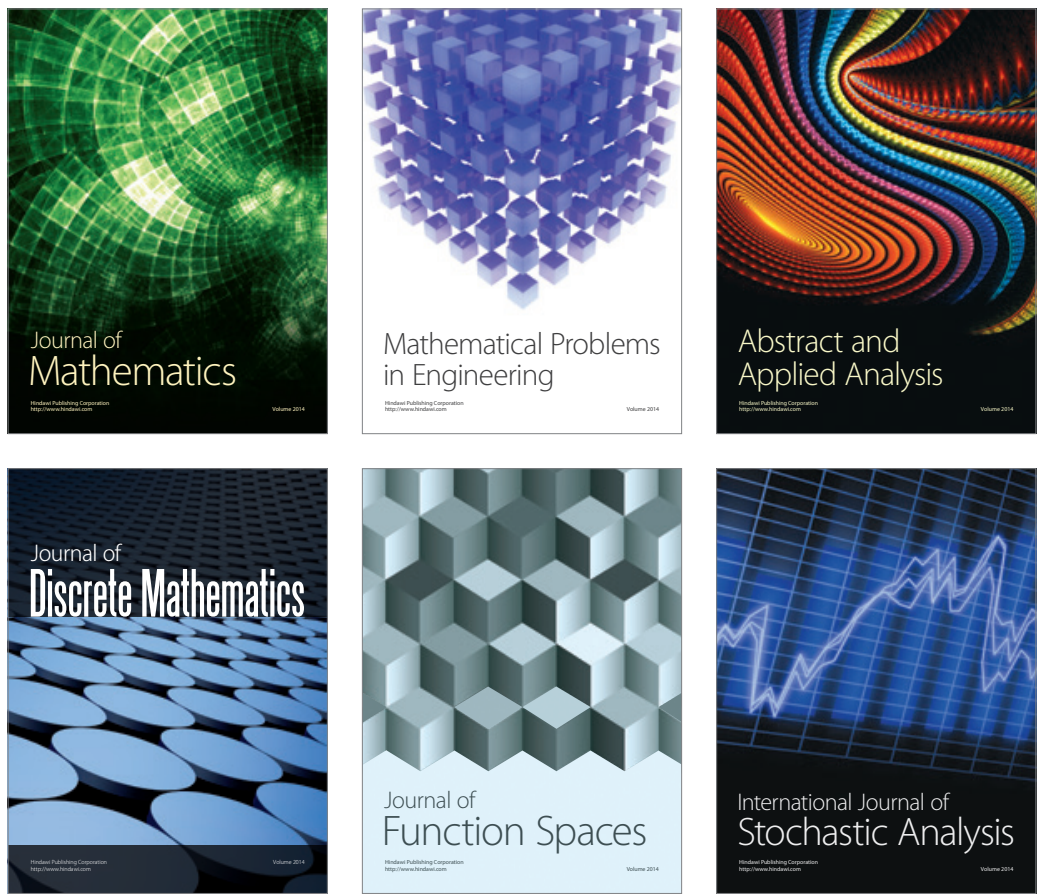

Journal of

Function Spaces

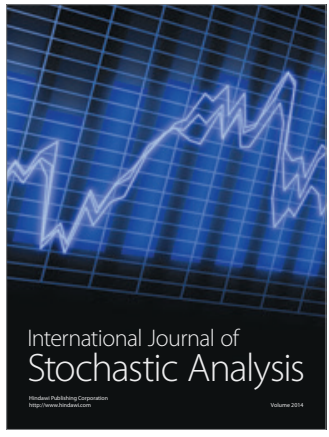

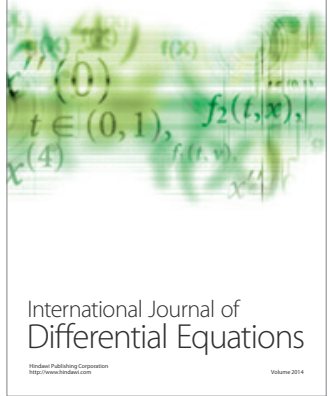
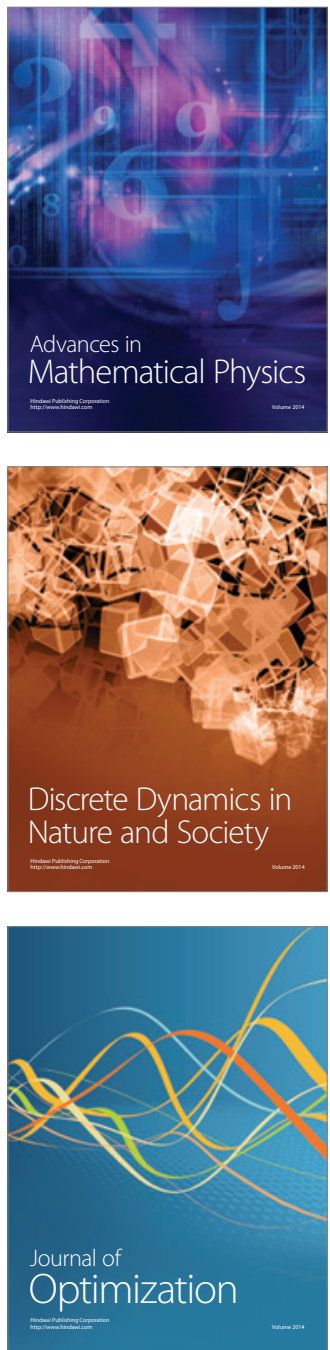